\title{
Advances in the management of gout: Critical appraisal of febuxostat in the control of hyperuricemia
}

This article was published in the following Dove Press journal: International Journal of Nephrology and Renovascular Disease 8 February 2010

Number of times this article has been viewed

\author{
Lada Beara-Lasic ${ }^{1,3}$ \\ Michael H Pillinger ${ }^{2,3}$ \\ David S Goldfarb ${ }^{1,3}$ \\ 'Divisions of Nephrology and \\ ${ }^{2}$ Rheumatology, Department of \\ Medicine, NYU Langone Medical \\ Center, New York, NY, USA; \\ ${ }^{3}$ Department of Medicine, New York \\ Harbor VA Health Care System, \\ New York, NY, USA
}

\begin{abstract}
Gout recently passed rheumatoid arthritis to become the most common inflammatory arthritis in the United States (US). However, epidemiologic studies indicate that the quality of gout management is suboptimal owing to both patient and physician issues. Only three options for urate-lowering therapy are currently available in the US: allopurinol, probenecid, and recently, febuxostat. Probenecid is generally safe except for the occurrence of urolithiasis, but is only effective for the subset of patients with better kidney function. Allopurinol use is limited due to its side effects, potential toxicity of uncertain magnitude in patients with renal disease, and failure to achieve targeted serum urate levels. In part this failure may be due to the necessity for it to be titrated for optimal therapeutic effect. Febuxostat is a new medication that may offer several advantages and can be given as an alternative to allopurinol. We review the basic biology and clinical performance of febuxostat, and consider the potential utility of this agent in comparison to the older, better-established gout therapeutics.
\end{abstract}

Keywords: allopurinol, gout suppressants, nephrolithiasis, uric acid, urolithiasis

\section{Introduction}

The incidence of gout is on the rise, and gout recently passed rheumatoid arthritis to become the most common inflammatory arthritis in the United States, affecting some three million individuals. ${ }^{1,2}$ In gout, the presence of high levels of serum urate (hyperuricemia) results in the precipitation of monosodium urate crystals, which then activate the complement cascade, macrophages and neutrophils to stimulate episodic and often severe inflammatory responses. ${ }^{3,4}$ The deposition of large masses of sodium urate crystals in the synovium, surrounded by coronas of inflammatory cells (tophaceous gout), causes tissue and joint damage and chronic pain. Moreover, recent studies have identified associations between gout and multiple co-morbidities, including hypertension, diabetes, obesity, and coronary artery disease, with some evidence that gout or hyperuricemia may contribute to these conditions. ${ }^{5-7}$

Management of gout generally requires the use of a combination of anti-inflammatory and urate-lowering agents, including colchicine, nonsteroidal anti-inflammatory drugs (NSAIDs) and glucocorticoids (anti-inflammatory), as well as probenecid and allopurinol (urate-lowering). While these urate-lowering agents are physiologically effective (with recommendations from the European League Against Rheumatism suggesting that a target serum urate $\leq 6.0 \mathrm{mg} / \mathrm{dL}$ is optimal to reduce attacks ${ }^{8}$ ), studies indicate that the quality of gout management is typically poor, owing to both patient and physician issues. ${ }^{9}$ Moreover, the high rate of co-morbidities found in gout patients frequently translate into contraindications
Correspondence: Lada Beara-Lasic Nephrology Section/ I I G, New York DVAMC, 423 E. 23 St., New York, NY I00I0, USA

$\mathrm{Tel}+\mathrm{I} 2126867500 \times 3876$

Fax + I $21295 \mid 6842$

Email lada.beara-lasic@va.gov 
to the currently available therapies. ${ }^{10}$ Two other agents may have limited use. Benzbromarone (an effective uricosuric) has been largely withdrawn from the market owing to hepatotoxicity but is still available in some European countries. ${ }^{11}$ Rasburicase (recombinant urate oxidase) degrades urate, but its cost, allergenicity and tendency to tachyphylaxis limit its effectiveness; it has been approved for tumor lysis syndrome but not gout. ${ }^{12}$ Accordingly, newer, better and safer medications are still needed.

Uric acid is an end-product of purine metabolism in humans and other mammalian species, and is the result of the step-wise actions of the enzyme xanthine oxidase/xanthine dehydrogenase, which converts the purine breakdown product hypoxanthine (via xanthine) to uric acid. ${ }^{13}$ Inhibition of xanthine oxidase/xanthine dehydrogenase by the purine analog allopurinol results in decreased serum uric acid levels and improved prognosis for gout. However, some individuals may not tolerate allopurinol owing to a potentially lethal hypersensitivity syndrome. ${ }^{14}$ Since allopurinol and its active metabolite are excreted by the kidneys, some controversy exists as to the safety of allopurinol in patients with chronic kidney disease (CKD). Moreover, some patients fail to achieve sufficient urate lowering with the use of allopurinol. Febuxostat (also known as TEI-6720, TMX-67 and Uloric ${ }^{\mathrm{TM}}$; Takeda Pharmaceuticals, Deerfield, IL) is an orally-administered inhibitor of xanthine oxidase that was approved for the treatment of chronic hyperuricemia and gout in 2008 in Europe and in 2009 in the US. Febuxostat has a number of biochemical and pharmacodynamic differences from allopurinol, suggesting that, in some patients at least, it may represent a better option for serum urate management. In this manuscript, we review the basic biology and clinical performance of febuxostat, and consider the potential utility of this agent in comparison to the older, better-established gout therapeutics.

\section{Overview of pharmacology, mode of action, and pharmacokinetics of febuxostat \\ Basic pharmacology}

Like allopurinol, febuxostat (2-(3-cyano-4-isobutoxyphenyl)4-methylthiazole-5-carboxylic acid; Figure 1) inhibits xanthine oxidase (XO), an enzyme with a molybdenum-containing active site, to reduce the production of uric acid from hypoxanthine. Activity of XO depends on the redox state of its catalytic center molybdenum, complexed by a pterin compound to form the molybdenum-pterin cofactor. However, the mechanisms of action of these two XO inhibitors are not identical. Allopurinol is a purine analogue and is metabolized both by XO

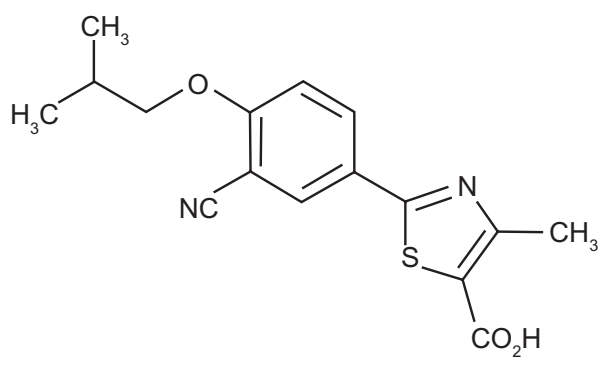

Figure I Chemical structure of febuxostat (Uloric ${ }^{\circledR}$ package insert; Takeda Pharmaceuticals America, Deerfield, IL).

and by the phosphoribosyltransferases, hypoxanthine-guanine phosphoribosyltransferase (HGPRT) and orotate phosphoribosyltransferase (OPRT), to form nucleotide analogues. Allopurinol exerts relatively weak competitive inhibition of the oxidized form of $\mathrm{XO}$, which rapidly oxidizes allopurinol to oxypurinol. In turn, oxypurinol binds tightly to, and inhibits, the reduced form of $\mathrm{XO}$, resulting in suicide inhibition of $\mathrm{XO}$ activity. In contrast, oxypurinol binds only weakly to the oxidized form of $\mathrm{XO}$, and is displaced from the reduced form of the enzyme during spontaneous re-oxidation of molybdenum, with subsequent $\mathrm{XO}$ re-activation.

In contrast to allopurinol, febuxostat is not a purine analogue. Febuxostat's inhibition of XO is mediated by highaffinity binding of febuxostat within the narrow XO molecular channel leading to the molybdenum-pterin active site. In binding to this channel, febuxostat blocks substrate access to the active site independent of the redox-state of the molybdenum cofactor. It exerts a potent mixed-type inhibition, inhibiting both the oxidized and reduced forms of the $\mathrm{XO} \cdot{ }^{15}$ Febuxostat does not inhibit other enzymes involved in purine and pyrimidine metabolism, which are inhibited by allopurinol and its metabolites due to their structural similarity to purines. ${ }^{16}$

In both in vitro and in vivo animal studies, febuxostat inhibited XO more potently than allopurinol. ${ }^{17}$ In one study in chimpanzees (which resemble humans in lacking uricase and having higher purine metabolite levels and urate excretion rates), both allopurinol and febuxostat (each $5 \mathrm{mg} / \mathrm{kg} /$ day orally), decreased serum urate levels after 24, 48 , and 72 hours of administration. However, febuxostat was more effective than allopurinol at lowering uric acid. Allopurinol decreased serum urate concentration by $28 \%(24 \mathrm{~h})$, $42 \%$ (48 h), and 45\% (72 h), as compared with the respective values for febuxostat of $56 \%, 70 \%$, and $74 \%{ }^{18}$

\section{Febuxostat pharmacokinetics}

Febuxostat's pharmacokinetics and pharmacodynamics have been studied in healthy volunteers as well as patients with 
renal or hepatic impairment, and in both nonblinded and randomized, placebo-controlled trials. ${ }^{19-22}$ Approximately $85 \%$ of febuxostat is absorbed after oral intake, with peak serum concentrations achieved after one hour in healthy subjects. ${ }^{21,23}$ Increasing the administered dose, or administering multiple doses, affects neither time to peak concentration $\left(\mathrm{t}_{\max }\right)$, nor tissue distribution of febuxostat. ${ }^{24}$ Maximum plasma concentration $\left(\mathrm{C}_{\max }\right)$ increases are dose-proportional over the $10-240 \mathrm{mg}$ dose range, and in the area under the plasma concentration-time curve (AUC) over the 10-120 mg range. ${ }^{19}$ Febuxostat in the blood is highly bound to plasma proteins, mainly albumin (99\%). At steady state, it has a low volume of distribution of about $0.7 \mathrm{~L} / \mathrm{kg}$, and a half life $\left(\mathrm{t}_{1 / 2}\right)$ of eight hours. ${ }^{23,24}$ Elimination half-life $\left(\mathrm{t}_{1 / 2}\right)$ values tended to increase with increasing doses and were longer after multiple doses compared with a single dose. ${ }^{19}$

Febuxostat is metabolized mainly by conjugation (via liver uridine diphosphate-glucuronosyltransferase (UGT) enzymes) into acylglucuronide metabolites (22\%-44\%), and to a lesser extent $(2 \%-8 \%)$ to its active oxidative metabolites 67M-1, 67M-2, and 67M-4 (via cytochrome P-450). ${ }^{21,25}$ Approximately $25 \%-45 \%$ of the drug is excreted in the urine as the conjugate, and an additional $2 \%-8 \%$ of the dose is excreted as oxidative metabolites. Less than $5 \%$ of a febuxostat dose is detected in the urine as unchanged drug. ${ }^{19,21}$

In otherwise healthy individuals, age and gender were found to have no significant effect on febuxostat's pharmacokinetics, pharmacodynamics, or safety after daily oral dosing with $80 \mathrm{mg}$. These data suggested that no dose adjustment should be necessary based on these parameters. ${ }^{24}$

\section{Febuxostat and renal impairment}

Since patients with gout often have varying degrees of CKD, several studies have examined the pharmacokinetics and pharmacodynamics of febuxostat in the setting of renal impairment. In a phase I trial, Hoshide determined the AUC of unchanged febuxostat in the plasma of 15 patients, assigned to 3 equal groups according to their renal function. ${ }^{26}$ Normal renal function was defined as creatinine clearance $(\mathrm{CrCl}) \geq 80 \mathrm{~mL} / \mathrm{min}$, mild renal impairment as $\mathrm{CrCl} 50-80 \mathrm{~mL} / \mathrm{min}$, and moderate renal impairment as $\mathrm{CrCl} 30-50 \mathrm{~mL} / \mathrm{min}$. Febuxostat was administered as a single dose of $20 \mathrm{mg}$ orally within 30 minutes after breakfast. Although increased plasma AUC for unchanged febuxostat was observed in the moderately renally- impaired group (4005.26 $\pm 1467.4 \mathrm{ng} \cdot \mathrm{h} / \mathrm{ml}$ vs the groups with mild renal impairment $(2338.62 \pm 290.36 \mathrm{ng} \cdot \mathrm{h} / \mathrm{ml})$ and normal function $(2644.08 \pm 593.04 \mathrm{ng} \cdot \mathrm{h} / \mathrm{ml}))$, the difference was less than twofold. On this basis, the authors concluded that moderate renal dysfunction did not have a significant impact on febuxostat metabolism. Five mild adverse events (AEs) were reported: gastric ulcer, constipation, headache, nausea, and upper respiratory tract infection; of these, only nausea was considered to be possibly related to febuxostat. It was not specified how the AEs were distributed according to kidney function. ${ }^{26}$

A second, larger phase I trial to evaluate safety, pharmacokinetics and pharmacodynamics of febuxostat in subjects with renal impairment was conducted as a parallel-group, open-labeled, multiple-dose study in three study centers. ${ }^{21}$ 32 men and women aged between 26 and 76 years were divided into four groups based on $\mathrm{CrCl}$ assessed by 24-hour urine collection. Normal renal function was defined as $\mathrm{CrCl}>80 \mathrm{~mL} / \mathrm{min} / 1.73 \mathrm{~m}^{2}(\mathrm{n}=11)$, mild renal impairment as $\mathrm{CrCl} 50-80 \mathrm{~mL} / \mathrm{min} / 1.73 \mathrm{~m}^{2}(\mathrm{n}=6)$, moderate renal impairment as $\mathrm{CrCl} 30-49 \mathrm{~mL} / \mathrm{min} / 1.73 \mathrm{~m}^{2}(\mathrm{n}=7)$, and severe renal impairment as $\mathrm{CrCl} 10-29 \mathrm{~mL} / \mathrm{min} / 1.73 \mathrm{~m}^{2}$ $(\mathrm{n}=7)$. Febuxostat was administered in the $80 \mathrm{mg}$ dose once daily at approximately 8:00 AM, for seven consecutive days after an overnight fast. Mean febuxostat $\mathrm{AUC}_{24, \mathrm{u}}$ (estimated as the product of the AUC and the fraction of unbound febuxostat in plasma) and terminal phase elimination halflife $\left(t_{1 / 2 z}\right)$ values tended to increase with increasing renal impairment, whereas mean febuxostat $\mathrm{t}_{\max }$ and $\mathrm{C}_{\text {max,u }}$ peak plasma concentration for unbound febuxostat $\left(\mathrm{C}_{\text {max,u }}\right)$ values were similar in subjects with CKD and those with normal renal function. Regression analysis indicated a statistically significant $(P \leq 0.05)$ inverse linear relationship of $\mathrm{CrCl}$ and $\mathrm{AUC}_{24, \mathrm{u}}$ and $\mathrm{t}_{1 / 2 \mathrm{z}}$. The urinary excretion of unchanged and total (unchanged + conjugated) febuxostat was lower in groups with greater degrees of renal impairment.

Mean renal clearance for febuxostat in subjects with mild, moderate, and severe renal impairment was approximately $48 \%, 46 \%$, and $77 \%$ lower than in those with normal renal function. Conversely, mean $\mathrm{C}_{\max }$ and $\mathrm{AUC}_{24}$ values for febuxostat active metabolites (67M-1, 67M-2, and 67M-4) were greater in subjects with renal impairment than in those with normal renal function. Regression analysis indicated significant linear relationship of $\mathrm{C}_{\text {max }}$ and $\mathrm{AUC}_{24}$ values for $67 \mathrm{M}-1$, 67M-2, and 67M-4, except $\mathrm{C}_{\text {max }}$ of 67M-1. Renal clearance of the metabolites was lower for subjects in the renal impairment groups. There were no reported deaths, serious adverse events (SAEs) or premature discontinuations due to AEs during this study. The majority of AEs were mild or self-limiting, the most frequently reported being diarrhea, abdominal pain, headache and flushing. When analyzed according to renal function, the frequency 
of AEs was comparable across groups, with a tendency to more headaches and flushing in the normal renal function group and diarrhea in the severe and moderate renal impairment group. The mean percent decrease in serum uric acid between the normal and the impaired renal function groups was comparable. Although plasma exposure to febuxostat and its metabolites was significantly higher in the renal impairment groups, the authors suggested that since the drug was well-tolerated, dose adjustment for renal function did not appear to be necessary. Mean serum concentrations of xanthine did increase substantially compared with baseline after seven days, which could raise concerns for the potential of xanthine crystal formation. However, the concentrations of serum xanthine were substantially lower than the solubility limit of xanthine in the serum, while the urinary concentration of xanthine did not increase with renal impairment. Overall, the authors concluded that the $80 \mathrm{mg}$ febuxostat dose does not require adjustment in $\mathrm{CKD} .^{21}$

\section{Febuxostat and hepatic impairment}

The effects of mild-to-moderate hepatic impairment on pharmacokinetics, pharmacodynamics, and safety of febuxostat were studied in a phase I study. ${ }^{20}$ Subjects took $80 \mathrm{mg}$ of oral febuxostat daily for seven days. There were no statistically significant differences in the plasma pharmacokinetic parameters for unbound febuxostat and its active metabolites among three groups: eight subjects with mild hepatic impairment (Child-Pugh class A), eight subjects with moderate hepatic impairment (Child-Pugh class B), and 11 subjects with normal hepatic function. The percentage decrease in serum uric acid was lower in hepatic impairment groups (49\% with mild liver disease and 48\% with moderate liver disease) compared to the normal hepatic group (62\%). This lower percentage decrease was not considered clinically significant. The safety and efficacy of febuxostat in severe hepatic impairment has not been studied. However, hepatic toxicity has not been reported in animal studies.

\section{Febuxostat safety and tolerability}

Toxic effects of allopurinol are most typically seen in the setting of the allopurinol sensitivity syndrome, a rare but occasionally life-threatening condition. It has been suggested that febuxostat, by virtue of its greater selectivity, its nonpurine structure and its lesser reliance on renal excretion, may have an improved safety profile when compared with allopurinol. While this has not been demonstrated in any head-to-head study, safety profiles to date do suggest that febuxostat is generally safe and well-tolerated.
In a multicenter, randomized, double-blind, placebo-controlled, phase III trial involving 153 patients, the safety profile of febuxostat in patients with gout was evaluated. ${ }^{27}$ Patients with serum creatinine $>1.5 \mathrm{mg} / \mathrm{dL}(\mathrm{CrCl}<50 \mathrm{~mL} / \mathrm{min})$ were excluded. Subjects received febuxostat $40 \mathrm{mg}, 80 \mathrm{mg}$, and $120 \mathrm{mg}$ or placebo once daily for 28 days. There were no significant differences between febuxostat and placebo groups with regard to treatment-related AEs, the majority of which were mild to moderate in severity and dose-independent. The most frequent side effects were diarrhea, abnormal liver function tests and abdominal pain. The only possible SAE was Guillain-Barré syndrome in one patient who was taking $80 \mathrm{mg}$ of febuxostat.

In FACT, a phase III, randomized, double-blind trial, 762 patients were randomly assigned to febuxostat (80 or $120 \mathrm{mg}$ ) or allopurinol (300 mg once daily) for 52 weeks. ${ }^{28}$ Patients with serum creatinine $>1.5 \mathrm{mg} / \mathrm{dL}(\mathrm{CrCl}<50 \mathrm{~mL} / \mathrm{min})$ were excluded. Overall incidence of treatment-related AEs was similar in all three groups, and included abnormal liverfunction tests, diarrhea, headaches, joint-related signs and symptoms and musculoskeletal and connective-tissue signs and symptoms. Once again, most side effects were mild to moderate in severity. Discontinuation rates were significantly higher in the $120 \mathrm{mg}$ febuxostat group, but were similar in $80 \mathrm{mg}$ febuxostat group and allopurinol group. There were four deaths in the febuxostat groups and none in the allopurinol groups but the difference was not statistically significant $(P=0.31)$.

APEX (Allopurinol- and Placebo-Controlled, Efficacy Study of Febuxostat) a phase III, randomized, double-blind, placebo-controlled, parallel group trial included a small subset of subjects with moderate CKD (serum creatinine $>1.5$ to $\leq 2 \mathrm{mg} / \mathrm{dL}$ ). ${ }^{29}$ The trial included 1072 subjects who were randomized to once-daily febuxostat $(80 \mathrm{mg}, 120 \mathrm{mg}$, or $240 \mathrm{mg}$ ), allopurinol (100 mg or $300 \mathrm{mg}$ based on renal function) or placebo, followed for 28 weeks. The frequency of adverse events was similar across the treatment groups. There were statistically increased incidences of diarrhea and dizziness in the febuxostat $240 \mathrm{mg}$ group compared to the other treatment groups. There were no deaths reported during the study and the frequency of SAEs was similar in all groups. The most SAEs observed in all treatment groups were cardiovascular disorders (chest pain, coronary artery disease, myocardial infarction, and atrial fibrillation), in patients with a history of underlying cardiovascular disease or risk factors. These SAEs led to withdrawal in one patient taking $80 \mathrm{mg}$ of febuxostat and two patients taking $120 \mathrm{mg}$ of febuxostat. Overall nine patients withdraw from the study 
due to serious AEs, one patient from the allopurinol group, and eight patients from the febuxostat treatment groups. However, only one subject experienced an SAE that was considered by the investigator to be related to the study drug. The subject reported a history of kidney stones and experienced a gradual increase in serum creatinine while receiving $240 \mathrm{mg}$ of febuxostat from $1.1 \mathrm{mg} / \mathrm{dL}$ to $1.5 \mathrm{mg} / \mathrm{dL}$ on day 197. The serum creatinine returned to $1.3 \mathrm{mg} / \mathrm{dL}$ during treatment with febuxostat $120 \mathrm{mg}$ in a subsequent extension trial. ${ }^{29}$ AEs leading to withdrawal occurred in 78 subjects with similar frequency in all treatment groups. The most frequent AEs leading to withdrawal were abnormal liver function tests and diarrhea.

In EXCEL, an open-label extended comparative treatment trial, 1086 patients were assigned to febuxostat $80 \mathrm{mg}$ or $120 \mathrm{mg}$ or allopurinol $300 \mathrm{mg}$ or $100 \mathrm{mg}$ and were followed for 40 months. ${ }^{30} \mathrm{~A}$ subset of patients with serum creatinine between 1.5 and $2 \mathrm{mg} / \mathrm{dL}$ were included. Patients were permitted reassignment to achieve uric acid levels $<6 \mathrm{mg} / \mathrm{dL}$. Consequently, the duration of exposure was longer on febuxostat than on allopurinol. AEs were adjusted to duration of exposure; when adjusted, similar rates of AEs and SAEs, as well as rates of AEs resulting in premature discontinuation, were reported. The most frequent SAEs were cardiac disorders which were equally represented in all treatment groups, when adjusted for duration of exposure. All 10 deaths reported (six were related to cardiovascular disease) occurred among subjects receiving febuxostat, with no dose- or timerelation. There is no clear mechanism to explain these events. A need for further evaluation is suggested. ${ }^{30}$

In FOCUS (Febuxostat Open-label Clinical trial of Urate-lowering efficacy and Safety), the 28-day Phase II trial described above was extended into a five-year open-label trial. ${ }^{31}$ Of 153 subjects enrolled in the initial study, 116 were enrolled in the extension study. All subjects were initially started on $80 \mathrm{mg}$ daily febuxostat for four weeks which was then adjusted to 40,80 , or $120 \mathrm{mg}$ to maintain serum uric acid between 3 and $<6 \mathrm{mg} / \mathrm{dL}$, to respond to an adverse event, or at the discretion of the investigator. The majority of AEs were reported to be mild to moderate and investigators considered all SAEs unrelated to study drug. However, there was no placebo or comparative treatment group to assess the difference. Serious AEs were reported by 21/116 subjects; the most frequent SAE was atrial fibrillation and it was experienced by five subjects while on febuxostat $80 \mathrm{mg} /$ day. Thirteen subjects discontinued treatment secondary to AEs. The most common AEs that caused withdrawal from the study were abnormal liver function tests, $\mathrm{n}=3$; cancers, $\mathrm{n}=2$; and two had increased serum creatinine. All three elevated liver enzymes and one increased creatinine were considered by investigators to be related to study drug.

Based on these and other observations, the prescribing information for febuxostat recommends monitoring liver function tests intermittently, and monitoring for signs and symptoms of myocardial infarction and stroke. Since febuxostat (like allopurinol) may increase serum levels of agents that are otherwise metabolized by xanthine oxidase, and so induce secondary toxicity (potentially fatal aplasia), the use of febuxostat in patients taking azathioprine, as well as mercaptopurine and theophylline, is contraindicated. No dosing adjustment is recommended in patients with mild to moderate renal impairment, mild to moderate hepatic impairment, or in geriatric patients (see Uloric package insert).

\section{Efficacy studies Febuxostat efficacy in patients with hyperuricemia and gout}

The above-described phase II and phase III trials were also designed to assess various aspects of febuxostat's efficacy. In the 28-day phase II trial, patients received colchicine for two weeks before, and two weeks after starting either febuxostat $\left(40,80\right.$, or $120 \mathrm{mg}$ once daily) or placebo. ${ }^{27}$ The primary efficacy endpoint in this study was the proportion of subjects in each treatment group with serum uric acid levels $<6.0$. By the end of the study significantly greater proportions of patients in the febuxostat groups had achieved this endpoint relative to placebo, and the mean percentage reductions in serum uric acid from baseline were greater than placebo in all febuxostat groups, with the greatest reductions in patients receiving $120 \mathrm{mg} /$ day of febuxostat (reduction of $53 \%-59 \%$ in serum urate). Significantly greater proportions of patients in the febuxostat groups also achieved lower serum urate targets $(<4.0$ and $<3.0 \mathrm{mg} / \mathrm{dL})$. Thus, febuxostat at all doses was superior to placebo at lowering serum urate levels. Perhaps not surprisingly, patients with the highest baseline levels of serum urate in each group were least likely to achieve the target urate in any of the therapy arms. In patients on colchicine, the incidence of gouty flares was not different among the four groups (range $8 \%-13 \%$ ). However, in patients no longer on colchicine, the incidence of gouty flares was higher in the febuxostat 80 and $120 \mathrm{mg} /$ day groups ( $40 \%$ and $42 \%$, respectively) than in the placebo $(34 \%)$ or febuxostat $40 \mathrm{mg} /$ day (30\%) groups, consistent with the established observation that urate lowering by any means acutely increases the risk for gout flare in the absence of inflammatory prophylaxis. 
In the FACT trial (febuxostat 80 or $120 \mathrm{mg}$ /day vs allopurinol for 52 weeks), patients received prophylaxis against gout flares for the first eight weeks of the study, using either colchicine or naproxen. ${ }^{32}$ The primary efficacy endpoint was a serum urate concentration $<6.0 \mathrm{mg} / \mathrm{dL}$ at each of the last three monthly visits, with secondary endpoints including proportion of subjects with a serum urate $<6.0 \mathrm{mg} / \mathrm{dL}$ at each visit, and the percentage reduction in serum urate from baseline at each visit. Clinical endpoints included reduction of incidence in gout flares and (for patients with tophi) reduction in tophus number and area. The primary end point was achieved by $53 \%$ of patients taking $80 \mathrm{mg}$ febuxostat, $62 \%$ taking $120 \mathrm{mg}$, and only $21 \%$ taking allopurinol. Secondary endpoints of urate lowering were also achieved more frequently in the febuxostat groups, and (in a post hoc analysis) patients in both febuxostat groups also achieved lower targets of serum urate $(5.0$ and $4.0 \mathrm{mg} / \mathrm{dL})$ more frequently than those in the allopurinol group. During the initial induction phase, the incidence of gouty attacks was significantly greater in the febuxostat $120 \mathrm{mg}$ group than in the febuxostat $80 \mathrm{mg}$ or allopurinol $300 \mathrm{mg}$ group, but these differences were not observed during the remainder of the study. All three groups experienced a transient surge in gout flares, lasting until approximately week 20, upon withdrawal of the initial inflammatory prophylaxis. In spite of its superiority at lowering serum urate levels, febuxostat showed a trend, but no statistically significant superiority to allopurinol in preventing gout flares by 52 weeks. Patients taking febuxostat also demonstrated a nonstatistically significant trend toward improved reduction in tophus surface area $(83 \%$ reduction for febuxostat $80 \mathrm{mg}$ and $66 \%$ reduction for febuxostat $120 \mathrm{mg}$, vs $50 \%$ reduction for patients taking allopurinol $300 \mathrm{mg}$ ). Overall, the authors concluded that febuxostat at all doses was superior to allopurinol $300 \mathrm{mg}$ for urate lowering.

The primary efficacy end point of the APEX trial (febuxostat 80,120 , and $240 \mathrm{mgs}$ vs allopurinol $300 \mathrm{mg}$; for patients with serum creatinine level $1.5-2.0 \mathrm{mg} / \mathrm{dL}, 100 \mathrm{mg}$ daily vs placebo) was the proportion of subjects with the last three monthly serum urate levels $<6.0 \mathrm{mg} / \mathrm{dL} .{ }^{29}$ Secondary end points were similar to those in FACT and included proportion of subjects with a serum urate $<6.0 \mathrm{mg} / \mathrm{dL}$ at each visit, percent reduction in serum urate, proportion of subjects requiring treatment for a gout flare, and reduction in size and number of tophi. A significantly greater percentage of patients receiving any dose of febuxostat achieved the primary end point vs allopurinol, with $50 \%-70 \%$ of the febuxostat, $20 \%-30 \%$ of the allopurinol, and none of the placebo subjects achieving target levels. By the time of the last visit, $76 \%$ of febuxostat $80 \mathrm{mg} /$ day subjects, $87 \%$ of febuxostat $120 \mathrm{mg} /$ day subjects, and $94 \%$ of febuxostat $120 \mathrm{mg} /$ day patients had achieved serum urate levels $<6.0$, compared with $41 \%$ of the allopurinol subjects and $1 \%$ of the placebo group. Febuxostat was also more effective than both allopurinol and placebo in urate lowering in patients with high ( $\geq 10 \mathrm{mg} / \mathrm{dL}$ ) baseline urate levels. As in the FACT trial, a greater proportion of patients in the higher dose (120 and $240 \mathrm{mg}$ ) febuxostat groups experienced gouty flares during the initiation of the study, whereas no differences between the febuxostat and allopurinol groups were seen at longer time points. Among patients with tophi, treatment with febuxostat $120 \mathrm{mg}$, but, not with other doses of either febuxostat or allopurinol, resulted in a statistically significant decrease in the number of tophi versus placebo at week 28 . In subjects with renal impairment, the use of allopurinol $100 \mathrm{mg} /$ day was not sufficient to achieve urate lowering.

The EXCEL trial was longer than any of the previous trials, and represented a combined extension study of patients who had previously enrolled in either FACT or APEX. 1280 patients were screened, and 1086 were enrolled and followed for up to 40 months, permitting assessment of long-term urate lowering on the incidence of gouty flares and the presence of tophi. Although the protocol was changed slightly after initiation, patients received either febuxostat $(80 \mathrm{mg}$ or $120 \mathrm{mg} /$ day) or allopurinol (300 mg/day; $100 \mathrm{mg}$ for a small number of patients with renal impairment); some provision was permitted for changing medications for lack of efficacy. The primary efficacy endpoint was the proportion of subjects with serum urate $<6.0 \mathrm{mg} / \mathrm{dL}$ at each visit; secondary endpoints included reduction of incidence of gout flares, percentage reduction in number of tophi, and reduction in size or disappearance of an index tophus. In the first months of treatment, patients in the febuxostat $80 \mathrm{mg}$ and $120 \mathrm{mg}$ groups achieved target urate lowering more often than those in the allopurinol group. For example, after one month, $81 \%$ of the febuxostat $80 \mathrm{mg}$ subjects, and $87 \%$ of the febuxostat $120 \mathrm{mg}$ subjects achieved serum urate $<6.0 \mathrm{mg} / \mathrm{dL}$, versus $49 \%$ of the allopurinol $300 \mathrm{mg}$ subjects. Beyond 20 months, all three groups achieved a similar frequency of urate lowering, a phenomenon the authors ascribed to the shift of some allopurinol patients, for whom the drug had been inadequate, into the febuxostat groups. Regarding gout flares, by the end of the study all three groups achieved a similar reduction in gout flares, but again, the shift of some allopurinol "failures" to the febuxostat groups made it impossible to compare the relative efficacies of the drugs overall at these late time points. 
As in prior studies, treatment in all three urate-lowering arms led to an increase in gouty attacks during the initial urate-lowering phase (4-6 months), with the higher dose of febuxostat $(120 \mathrm{mg})$ producing the greatest increase in gout flares. Patients in all three groups demonstrated reduction in size and number of tophi, consistent with the long-term lowering of uric acid to similar degrees.

Finally, the open-label FOCUS trial (febuxostat $80 \mathrm{mg} /$ day, with an option to adjust dosing in an initial phase to 40 or $120 \mathrm{mg}$ ) had no control group, and took as its primary end point the proportion of subjects achieving serum urate $<6.0 \mathrm{mg} / \mathrm{dL} .{ }^{31}$ Secondary endpoints included percent reduction from baseline serum urate, proportion of subjects achieving a urate of $<5.0$ and $<4.0 \mathrm{mg} / \mathrm{dL}$, the proportion of subjects who required treatment for gout flare and the proportion of subjects with resolution of tophi. Most patients completed the study on $80 \mathrm{mg}$ daily, though a smaller number remained on $40 \mathrm{mg}$ or $120 \mathrm{mg}$ after the initial adjustment period. By the end of the study period, between 80 and $100 \%$ of patients had achieved the target urate value. Despite the generally fast effect of febuxostat, some patients required as long as 3-4 years to achieve their lowest urate levels. Overall, the percentage of patients experiencing gouty flares initially increased, then gradually declined, approaching zero by the end of the study period. Approximately $70 \%-80 \%$ of patients experienced complete resolution of an index tophus. The rate of tophus disappearance was relatively linear until week 208, after which no additional tophus resolution was seen.

Collectively, these trials attest to the ability of febuxostat to rapidly reduce serum urate. In this regard, febuxostat at all doses appeared to be more effective than allopurinol $300 \mathrm{mg}$ (or $100 \mathrm{mg}$ in patients with mild to moderate renal failure). However, in this regard it is worth noting that the FDA-approved dose range for allopurinol is up to $800 \mathrm{mg}$, and no study has compared febuxostat with higher- or sliding-dose allopurinol. The ability of febuxostat to lower urate is clearly reflected in its ability to eventually reduce gouty flares, although its relative benefit in this regard vs allopurinol (300 mg or any other dose) is not clearly established by the studies to date. Finally, these studies indicate that successful long-term reduction of serum urate, whether by febuxostat or allopurinol, eventually results in the potential for almost complete abrogation of gout flares as well as the resolution of previously established tophi.

\section{Febuxostat and renal stones}

Hyperuricosuria is a risk factor for formation of both uric acid and calcium stones. However, lowering uric acid excretion is not the preventive strategy of choice for uric acid stones and has an adjunctive role for prevention of calcium stones. Risk factors for uric acid stones are low urine volume, hyperuricosuria, and most importantly, low urine $\mathrm{pH} .{ }^{33}$ The preeminent effect of low urine $\mathrm{pH}$ is demonstrated by the frequent occurrence of uric acid stones in patients who do not have hyperuricosuria but do have an unvaryingly low urine $\mathrm{pH}$, usually of 5.5 or less. The pathophysiology of the persistently low urine $\mathrm{pH}$ often seen in patients with gout now appears to be caused by insulin resistance, and accounts for a higher prevalence of uric acid stones in patients with the metabolic syndrome. ${ }^{34}$

While low urine $\mathrm{pH}$ and hyperuricosuria contribute to uric acid stones, in the absence of an acid urine, patients so affected are unlikely to have uric acid stones, and may have calcium stones. If hyperuricosuria is present in uric acid stone formers, treatment with urate-lowering therapy is unlikely to be effective in stone prevention if alkalinization of the urine is not achieved. Since even normal or low uric acid excretion may be associated with uric acid stone formation in an acid urine, as occurs in patients with ileostomies, urate-lowering therapy without alkalinization is unlikely to lower urine supersaturation to a value low enough to cause uric acid crystals to dissolve. Therefore the role of both allopurinol and febuxostat in uric acid stone formers is likely to be minimal. The drugs might best be applied in such patients who have difficulties achieving urine $\mathrm{pH}$ of 6.5 at least once a day or who persist in forming uric acid stones despite adequate alkalinization.

Hyperuricosuria may be a contributing risk factor for calcium stones. The evidence however is not conclusive as there is a discrepancy between the epidemiologic data and the results of a single randomized controlled trial. In three prospectively followed, well-studied cohorts of both men and women, increasing 24-hour uric acid excretion was not a predictor of a greater risk for stone incidence as compared with controls without stones. ${ }^{35}$ In fact, in younger women and men, uric acid excretion was inversely associated with stone incidence. These data are limited by not having information about stone composition, but the expectation would be that most stones were calcium.

The epidemiologic data contrast with the results of a study that demonstrated that allopurinol reduced the incidence of calcium stones. ${ }^{36}$ Patients with stones composed of more than $79 \%$ calcium oxalate, hyperuricosuria $(>800 \mathrm{mg} / \mathrm{dL}$ in men, $>750 \mathrm{mg} / \mathrm{dL}$ in women) and normocalciuria ( $<300 \mathrm{mg} / \mathrm{dL}$ in men, $<250 \mathrm{mg} / \mathrm{dL}$ in women) were eligible. Results for 60 patients were analyzed after randomization to either allopurinol $100 \mathrm{mg}$ three times a day, or placebo and up to three years of therapy. New stone events occurred in 
18 subjects receiving placebo and nine receiving allopurinol, a significant difference. The allopurinol group also had a significantly longer time before recurrence. Since allopurinol is not known to have any effect on calcium metabolism or urine chemistry other than lowering of uric acid excretion, the effect to reduce calcium stone formation is attributed to "salting out", the ability of uric acid to cause precipitation of dissolved calcium oxalate from solution. ${ }^{37}$

These interesting results suggest that febuxostat could have a role in preventing kidney stones. Its place for prevention of calcium stones could be determined by comparing its relative efficacy in lowering uric acid excretion in patients with calcium stones and if followed for long enough, its ability to prevent new stone events could be measured. Whether it is useful to lower uric acid excretion in patients with other abnormalities of urine chemistry, such as hypercalciuria or hypocitraturia, excluded from the allopurinol trial described above, would also be important to determine.

\section{Febuxostat from the patient perspective}

For gout patients who require urate lowering, only three options are currently available in the US: allopurinol, probenecid, and now febuxostat. In contrast to allopurinol and febuxostat, probenecid works to stimulate renal uric acid excretion by blocking renal tubular resorption of urate via inhibition of the URAT1 organic ion transporter. Probenecid is most effective in patients with normal glomerular filtration rates (GFR) who, as a result of intrinsic renal tubular defects, under-excrete uric acid. Probenecid has limited or no effect in patients with reduced GFR, whether due to chronic or acute kidney disease. Moreover, since probenecid increases uric acid delivery to the collecting system, it may raise the risk of calcium acid renal stones as described above. Consequently, even patients who do not have a history of renal stones require consumption of copious amounts of water or beverages daily to reduce stone risk. Given a history of uric acid stones, potassium or sodium citrate should be prescribed to alkalinize the urine. The relatively short half-life of probenecid requires that it be administered thrice daily. Therefore, although probenecid is a generally safe and effective drug, even the subset of patients for whom probenecid is a good choice tend to prefer an easier alternative.

For more than five decades, allopurinol has been that alternative. Most patients take allopurinol on a once-daily dosing schedule, and beyond anti-inflammatory prophylaxis, no ancillary medications are needed. In the majority of patients, allopurinol has little or no toxicity. However, a small number of patients develop the allopurinol hypersensitivity syndrome, which can involve liver, renal and marrow toxicity. For those patients - or for patients who develop a rash on allopurinol, since a rash may be a harbinger of the hypersensitivity syndrome - there are few if any good alternatives. Another drawback of allopurinol is the fact that many patients taking allopurinol fail to achieve targeted serum urate levels. In a spot study that we recently conducted, the mean serum uric acid level in patients receiving allopurinol from the primary care clinics of a large veterans hospital was $6.9 \mathrm{mg} / \mathrm{dL}$, nearly a point above the recommended maximum target serum urate level. ${ }^{38}$ The reasons for this failure of allopurinol are not fully elucidated, but most likely relate to the relatively limited efficacy of the $300 \mathrm{mg}$ dose; the failure of many physicians to appreciate that allopurinol should be titrated not to a specific dose, but to a serum urate target level; and the fact that in "real world medicine," the time required to titrate a patient to the necessary dose can sometimes be quite extended and provide ample opportunity for compliance failure. The superiority of febuxostat to allopurinol $300 \mathrm{mg}$ should not be construed as superiority to allopurinol overall, since allopurinol is US Food and Drug Administration (FDA)-approved at doses up to $800 \mathrm{mg} /$ day. On the other hand, patients who need such high levels of allopurinol will frequently take a long time to achieve the target dose, and will typically need to advance to twice-daily dosing.

For patients with CKD, controversy exists as to the safety of allopurinol, with some studies suggesting an increase in toxicity and others suggesting none, particularly when allopurinol dosing is started low and adjusted specifically to target urate (ie, to avoid prescribing more than is actually necessary). In general, the community consensus would appear to be moving to the latter point of view. ${ }^{39-41}$ It is likely, therefore, that adequate results can be achieved with allopurinol in renal patients, even those needing a higher dose; however, the safety profiles of such doses has not been well established. Moreover, the use of allopurinol in patients with renal insufficiency may require a slower titration period and more monitoring, with its attendant inconvenience to the patient. The fact that febuxostat is approved for individuals with mild to moderate renal insufficiency, defined as $\mathrm{CrCl}>30 \mathrm{~mL} / \mathrm{min}$, and does not need dosing adjustment in these individuals, makes for a simpler management algorithm for patients and physicians alike. It is important to note that all long term follow up studies excluded patients with creatinine higher than $2 \mathrm{mg} / \mathrm{dL}$. In addition, although elevation of creatinine has been reported as a very rare $\mathrm{AE}$, monitoring of kidney function is prudent. 
From the patient's point of view, therefore, febuxostat may offer several advantages. It can be given as an alternative to allopurinol for those patients who have allopurinol hypersensitivity; it will typically provide a more rapid timeto-target serum urate than allopurinol; its dosing is always once a day, and simplified by the fact that only two dose levels (40 $\mathrm{mg}$ and $80 \mathrm{mg}$ ) are available (and generally needed). When compared with the $300 \mathrm{mg}$ dose of allopurinol at least, febuxostat works better and faster to lower urate, and appears to be a better agent for reducing tophi. Patients who are less motivated to work with their physicians, have mild to moderate $\mathrm{CKD}$, or who need relief more quickly, may therefore benefit from the simplicity of febuxostat. On the other hand, it must be recognized that patients starting on febuxostat are more likely to experience a temporary increase in gouty flares than those starting allopurinol; the importance of rigorous anti-inflammatory prophylaxis therefore deserves emphasis. Given a possible slight increase in cardiac events, patients with cardiovascular disease should probably discuss this issue with their physicians. Moreover, to the extent that patients are also consumers, the relative costs of febuxostat vs generic allopurinol (US $\$ 161.40$ vs US $\$ 14.70$ for 30 days) will also warrant consideration. ${ }^{42}$

\section{Conclusions}

For more than 50 years, clinicians have treated gout without recourse to any new medications. Although the previously available drugs could be rendered effective in most patients, achieving an appropriate level of gout control has often been a long, slow affair, and many patients never achieved a desirable level of control. Given the limited alternatives, little attention was paid to the possible toxicities of available gout therapies, particularly in view of the fact that many patients harbored comorbidities that represent contraindications to treatment. Viewed in this light, febuxostat is a welcome alternative. At the very least, febuxostat use will be warranted in patients who require urate lowering but cannot tolerate allopurinol. The simplicity of febuxostat dosing, together with its established efficacy, may lead to its wider use, particularly among physicians with limited capacity to use probenecid or titrate allopurinol over time. With several other gout drugs currently in the pipeline, ${ }^{43-45}$ febuxostat may represent the first milestone in a new era of improved gout management.

\section{Disclosure}

Dr Beara-Lasic received funding from NIDDK and ORDR. Dr Goldfarb is on medical advisory boards for Takeda and
Sanofi-Aventis, consults for Watson, and has received research funding from NIDDK and Office of Rare Disease Research. Dr. Pillinger received educational funding from the American College of Rheumatology, research funding from the NIAMS and from the Arthritis Foundation, New York Chapter.

\section{References}

1. Terkeltaub R, Zelman D, Scavulli J, Perez-Ruiz F, Liote F. Gout Study Group: update on hyperuricemia and gout. Joint Bone Spine. 2009;76(4):444-446.

2. Lawrence RC, Felson DT, Helmick CG, et al. Estimates of the prevalence of arthritis and other rheumatic conditions in the United States. Part II. Arthritis Rheum. 2008;58(1):26-35.

3. Pillinger MH, Rosenthal P, Abeles AM. Hyperuricemia and gout: new insights into pathogenesis and treatment. Bull NYU Hosp Jt Dis. 2007;65(3):215-221.

4. Martinon F, Petrilli V, Mayor A, Tardivel A, Tschopp J. Gout-associated uric acid crystals activate the NALP3 inflammasome. Nature. 2006;440(7081): 237-241.

5. Annemans L, Spaepen E, Gaskin M, et al. Gout in the UK and Germany: prevalence, comorbidities and management in general practice 2000-2005. Ann Rheum Dis. 2008;67(7):960-966.

6. Krishnan E. Gout and coronary artery disease: epidemiologic clues. Curr Rheumatol Rep. 2008;10(3):249-255.

7. Heinig M, Johnson RJ. Role of uric acid in hypertension, renal disease, and metabolic syndrome. Cleve Clin J Med. 2006;73(12):1059-1064.

8. Zhang W, Doherty M, Bardin T, et al. EULAR evidence based recommendations for gout. Part II: Management. Report of a task force of the EULAR Standing Committee for International Clinical Studies Including Therapeutics (ESCISIT). Ann Rheum Dis. 2006;65(10): 1312-1324.

9. Singh JA, Hodges JS, Toscano JP, Asch SM. Quality of care for gout in the US needs improvement. Arthritis Rheum. 2007;57(5):822-829.

10. O'Brien WR, Keenan DB, Goldfarb DS, Pillinger MH. Prevalence of co-morbidities and relative contraindications to standard therapies in a cohort of gout patients. Arthritis Rheum. 58(12):3983.

11. Zurcher RM, Bock HA, Thiel G. Excellent uricosuric efficacy of benzbromarone in cyclosporin-A-treated renal transplant patients: a prospective study. Nephrol Dial Transplant. 1994;9(5):548-551.

12. Cammalleri L, Malaguarnera M. Rasburicase represents a new tool for hyperuricemia in tumor lysis syndrome and in gout. Int J Med Sci. 2007;4(2):83-93.

13. Wortmann R, Kelly WN. Gout and hyperuriciemia. In: Firestein GS, Budd RC, Harris EO, et al. editors. Kelley's Textbook of Rheumatology. 8th ed. Philadelphia, PA: Saunders Elsevier; 2008:1481-586.

14. Arellano F, Sacristan JA. Allopurinol hypersensitivity syndrome: a review. Ann Pharmacother. 1993;27(3):337-343.

15. Okamoto K, Eger BT, Nishino T, Kondo S, Pai EF, Nishino T. An extremely potent inhibitor of xanthine oxidoreductase. Crystal structure of the enzyme-inhibitor complex and mechanism of inhibition. $J$ Biol Chem. 2003;278(3):1848-1855.

16. Takano Y, Hase-Aoki K, Horiuchi H, et al. Selectivity of febuxostat, a novel non-purine inhibitor of xanthine oxidase/xanthine dehydrogenase. Life Sci. 2005;76(16):1835-1847.

17. Osada Y, Tsuchimoto M, Fukushima H, et al. Hypouricemic effect of the novel xanthine oxidase inhibitor, TEI-6720, in rodents. Eur J Pharmacol. 1993;241(2-3):183-188.

18. Komoriya K, Osada Y, Hasegawa M, et al. Hypouricemic effect of allopurinol and the novel xanthine oxidase inhibitor TEI-6720 in chimpanzees. Eur J Pharmacol. 1993;250(3):455-460.

19. Becker MA, Kisicki J, Khosravan R, et al. Febuxostat (TMX-67), a novel, non-purine, selective inhibitor of xanthine oxidase, is safe and decreases serum urate in healthy volunteers. Nucleosides Nucleotides Nucleic Acids. 2004;23(8-9):1111-1116. 
20. Khosravan R, Grabowski BA, Mayer MD, Wu JT, Joseph-Ridge N, Vernillet $\mathrm{L}$. The effect of mild and moderate hepatic impairment on pharmacokinetics, pharmacodynamics, and safety of febuxostat, a novel nonpurine selective inhibitor of xanthine oxidase. J Clin Pharmacol. 2006;46(1):88-102.

21. Mayer MD, Khosravan R, Vernillet L, Wu JT, Joseph-Ridge N, Mulford DJ. Pharmacokinetics and pharmacodynamics of febuxostat, a new non-purine selective inhibitor of xanthine oxidase in subjects with renal impairment. Am J Ther. 2005;12(1):22-34.

22. Khosravan R, Kukulka MJ, Wu JT, Joseph-Ridge N, Vernillet L. The effect of age and gender on pharmacokinetics, pharmacodynamics, and safety of febuxostat, a novel nonpurine selective inhibitor of xanthine oxidase. J Clin Pharmacol. 2008;48(9):1014-1024.

23. Bruce SP. Febuxostat: a selective xanthine oxidase inhibitor for the treatment of hyperuricemia and gout. Ann Pharmacother. 2006;40(12):2187-2194.

24. Khosravan R, Grabowski BA, Wu JT, Joseph-Ridge N, Vernillet L. Pharmacokinetics, pharmacodynamics and safety of febuxostat, a nonpurine selective inhibitor of xanthine oxidase, in a dose escalation study in healthy subjects. Clin Pharmacokinet. 2006;45(8):821-841.

25. Mukoyoshi M, Nishimura S, Hoshide S, et al. In vitro drug-drug interaction studies with febuxostat, a novel non-purine selective inhibitor of xanthine oxidase: plasma protein binding, identification of metabolic enzymes and cytochrome P450 inhibition. Xenobiotica. 2008;38(5):496-510.

26. Hoshide $\mathrm{S}$, Takahashi $\mathrm{Y}$, Ishikawa $\mathrm{T}$, et al. $\mathrm{PK} / \mathrm{PD}$ and safety of a single dose of TMX-67 (febuxostat) in subjects with mild and moderate renal impairment. Nucleosides Nucleotides Nucleic Acids. 2004;23(8-9): 1117-1118.

27. Becker MA, Schumacher HR Jr, Wortmann RL, et al. Febuxostat, a novel nonpurine selective inhibitor of xanthine oxidase: a twenty-eight-day, multicenter, phase II, randomized, double-blind, placebo-controlled, dose-response clinical trial examining safety and efficacy in patients with gout. Arthritis Rheum. 2005;52(3):916-923.

28. Becker MA, Schumacher HR Jr, Wortmann RL, et al. Febuxostat compared with allopurinol in patients with hyperuricemia and gout. $N$ Engl J Med. 2005;353(23):2450-2461.

29. Schumacher HR Jr, Becker MA, Wortmann RL, et al. Effects of febuxostat versus allopurinol and placebo in reducing serum urate in subjects with hyperuricemia and gout: a 28-week, phase III, randomized, doubleblind, parallel-group trial. Arthritis Rheum. 2008;59(11):1540-1548.

30. Becker MA, Schumacher HR, MacDonald PA, Lloyd E, Lademacher C. Clinical efficacy and safety of successful longterm urate lowering with febuxostat or allopurinol in subjects with gout. $J$ Rheumatol. 2009;36(6):1273-1282.

31. Schumacher HR Jr, Becker MA, Lloyd E, MacDonald PA, Lademacher C. Febuxostat in the treatment of gout: 5-yr findings of the FOCUS efficacy and safety study. Rheumatology (Oxford). 2009;48(2):188-194.
32. Becker MA, Schumacher HR Jr, Wortmann RL, et al. Febuxostat compared with allopurinol in patients with hyperuricemia and gout. N Engl J Med. 2005;353(23):2450-2461.

33. Maalouf NM, Cameron MA, Moe OW, Sakhaee K. Novel insights into the pathogenesis of uric acid nephrolithiasis. Curr Opin Nephrol Hypertens. 2004;13(2):181-189.

34. Maalouf NM, Cameron MA, Moe OW, ms-Huet B, Sakhaee K. Low urine $\mathrm{pH}$ : a novel feature of the metabolic syndrome. Clin J Am Soc Nephrol. 2007;2(5):883-888.

35. Curhan GC, Taylor EN. 24-h uric acid excretion and the risk of kidney stones. Kidney Int. 2008;73(4):489-496.

36. Ettinger B, Tang A, Citron JT, Livermore B, Williams T. Randomized trial of allopurinol in the prevention of calcium oxalate calculi. $N$ Engl J Med. 1986;315(22):1386-1389.

37. Ryall RL, Grover PK, Marshall VR. Urate and calcium stones-picking up a drop of mercury with one's fingers? Am J Kidney Dis. 1991;17(4): 426-430.

38. Keenan RT, Lehmann RA, O'Brien WR, Crittenden DB, Lee KH, and Pillinger MH. Gout management in primary care vs rheumatology: evidence for suboptimal treatment. Arthritis Rheum. 2009; 60(Suppl 10):1110.

39. Chao J, Terkeltaub R. A critical reappraisal of allopurinol dosing, safety, and efficacy for hyperuricemia in gout. Curr Rheumatol Rep. 2009;11(2):135-140.

40. Dalbeth N, Stamp L. Allopurinol dosing in renal impairment: walking the tightrope between adequate urate lowering and adverse events. Semin Dial. 2007;20(5):391-395.

41. Dalbeth N, Kumar S, Stamp L, Gow P. Dose adjustment of allopurinol according to creatinine clearance does not provide adequate control of hyperuricemia in patients with gout. $J$ Rheumatol. 2006;33(8): $1646-1650$.

42. Febuxostat (Uloric) for chronic treatment of gout. Med Lett Drugs Ther. 2009;51(1312):37-38.

43. Sundy JS, Becker MA, Baraf HS, et al. Reduction of plasma urate levels following treatment with multiple doses of pegloticase (polyethylene glycol-conjugated uricase) in patients with treatment-failure gout: Results of a phase II randomized study. Arthritis Rheum. 2008;58(9):2882-2891.

44. So A, De ST, Revaz S, Tschopp J. A pilot study of IL-1 inhibition by anakinra in acute gout. Arthritis Res Ther. 2007;9(2):R28.

45. Terkeltaub R, Sundy JS, Schumacher HR, et al. The interleukin 1 inhibitor rilonacept in treatment of chronic gouty arthritis: results of a placebo-controlled, monosequence crossover, non-randomised, singleblind pilot study. Ann Rheum Dis. 2009;68(10):1613-1617.

\section{Publish your work in this journal}

The International Journal of Nephrology and Renovascular Disease is an international, peer-reviewed open-access journal focusing on the pathophysiology of the kidney and vascular supply. Epidemiology, screening, diagnosis, and treatment interventions are covered as well as basic science, biochemical and immunological studies. The journal welcomes original

\section{Dovepress}

research, clinical studies, reviews \& evaluations, expert opinion and commentary, case reports and extended reports. The manuscript management system is completely online and includes a very quick and fair peerreview system, which is all easy to use. Visit http://www.dovepress.com/ testimonials.php to read real quotes from published authors. 